\title{
DON JUAN EN ITALIA EN EL SIGLO XVIII
}

Las amplias bibliografías sobre el tema de don Juan, en particular la más detallada de Armand E. Singer que se mantiene más o menos al día, incluyen referencias a unos libretos italianos del siglo XVIII anteriores al de Da Ponte (Praga, 1787) ${ }^{1}$. Desde luego, el que mejor se conoce es la obra de un acto atribuida a Giuseppe Bertati, Don Giovanni ossia Il convitato di pietra, para el cual compuso la música Giuseppe Gazzaniga. Esta obra se estrenó unos nueve meses antes de que apareciera Il Don Goivanni de Da Ponte-Mozart. En ella se encontrará la fuente de algunos pasajes de los cuales se sirvió Da Ponte, principalmente el famoso catálo$\mathrm{go}^{2}$. Lo que sabemos de otras piezas musicales no es siempre muy de fiar, ya que a veces se presentan dudas y confusiones en cuanto al libretista, compositor, título, fecha y lugar de estreno.

${ }^{1}$ Armand E. Singer, The Don Juan Theme, Versions and Criticism: A Bibliography, West Virginia University, Morgantown, 1965 y suplementos siguientes; Leo Weinstein, The Metamorphoses of Don Juan, Stanford University Press, Stanford, 1959; Everett W. Hesse, "Catálogo bibliográfico de Tirso de Molina (1648-1948), incluyendo una sección sobre la influencia del tema del Don Juan', EM, 5 (1949), 781-889; Stefan Kunze, Don Giovanni vor Mozart. Die Tradition der Don-Giovanni-Opera in italienischen Buffa-Theater des 18 Jahrhunderts, W. Fink, Munich, 1972. Esta obra de Kunze trata de todas las obras italianas de la década anterior a la ópera de Da Ponte-Mozart, inclusive la que se examina en este trabajo. Véanse esp. pp. 74-76 y 77. TAdDEo WIEL, I Teatri musicale Veneziani del Settecento. Catalogo delle opere in musica rappresentate nel secolo xviii in Venezia (1701-1800) con prefazione dell'autore, Fratelli Visentini, Venecia, 1897, reeditado en Leipzig, ed. Peters, 1979.

${ }^{2}$ En la Houghton Library de Harvard University se encuentra esta obra junto con L'impresario in angustie, probablemente de Giuseppe Maria Diodati, asignatura 1C7. D6206. 786 ig. La música es de Domenico Cimarosa. Véase infra n. 13. Sobre el catálogo ver D. LiDa, "Los catálogos de Don Giovanni y de Don Juan Tenorio", en CH. (3), pp. 553-561; y "The Catalogues of Don Giovanni and Don Juan Tenorio", HISt, 2 (1979), 64-86. 
Hace unos años tuvimos la buena suerte de poder examin: los textos de dos de esas obras que vienen interesando a los est diosos de la leyenda. Figuran en una colección de libretti de 1 . siglos XVII y XVIII que se encuentran en la Houghton Libra (Raros) de Harvard University ${ }^{3}$. Nuestra lectura podrá ayuda nos a aclarar algunas de las dudas que todavía persisten. La m. importante de las dos obras en cuestión es la de Nunziato Port Il convitato di pietra o sia Il dissoluto, "dramma tragicomico", pa: el cual compuso la música Vincenzo Righini, como verem pronto $^{4}$. Singer cataloga esta obra bajo el nombre de Righi con el título Don Giovanni ossia el convitato di pietra y considera qI se desconoce quién fue el libretista o que quizá haya sido A. c Filistri da Caramondani. Cree que el estreno tuvo lugar en Vi na el 21 de agosto de 1777. Se sugieren otros títulos (entre eli el nuestro citado con ligerísimas variantes de ortografía), otro 1 cal de estreno y otras fechas atribuidas (op. cit., p. 156, núr 1537). Leo Weinstein aprovecha los mismos datos (op. cit., 207, núm. 400).

Esto se debe cotejar con la cronología de Stefan Kunze q1 cita un estreno en Praga en 1777 en el Teatro Real de Kotze seguido ese mismo año de la representación del 21 de agosto । Viena. La edición de Praga no menciona ni el nombre del libr tista, ni al compositor; la de Viena únicamente incluye el c compositor. El nombre de Nunziato Porta sólo se encuentra el texto de Esterhazy, 1781, que se conserva en la Biblioteca $\mathrm{N}$

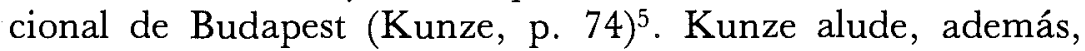
una posible representación anterior a todas las mencionadas, $\mathrm{q}$ puede haber tenido lugar en Praga en 1776. El texto de Houghton Library es el de una función en el Teatro Esterha de Viena durante el verano de $1781^{6}$. La ficha del catálogo c

${ }^{3}$ Me complace agradecer aquí la ayuda de Mollie della Terza, Ch Cataloguer de la Houghton, quien me llamó la atención sobre la obra de $\mathrm{P}$ ta, mientras catalogaba la colección de dramas italianos de los siglos XVI XVIII. Al repasar la lista completa, vi que incluía Il convito de F. Livigni, c también pude examinar y confirmar que la música es de Cimarosa. Ver nu tras pp. 710 ss. Se citan estas obras con permiso de la Houghton Libra Cambridge, Massachusetts.

${ }^{4}$ Asignatura 1C7. A100. B750, núm. 6 en el t. 64. Véase también $s$ GER, op. cit., p. 156.

5 "Der Name des Textdichters, Nunziato Porta, ist nur im Libretto 、 Esterhazy überliefert. In Prag scheint schon im Jahre 1776 ein Aufführ staatgefunden zu haben"' (p. 74).

${ }^{6}$ Singer y Weinstein sugieren fechas anteriores -1776 y 1779 , ader 
clara que la obra fue atribuida equivocadamente a Antonio de' Filistri da Caramondani ${ }^{7}$. En el texto, después del reparto, se lee: "La Musica è del Signor Vincenzo Righini. La Poesia è del Signor Nunziato Porta'.

El escrutinio del texto revela su deuda tanto a Tirso de Molina como a dramas italianos y a la commedia dell' arte, en particular en el personaje del criado que se llama Arlechino (sic). Es una comedia sostenuta de tres actos cuya acción se desarrolla en Castilla, nombre que se emplea en muchas obras extranjeras de la época para designar a toda España. La acción empieza al atardecer, mientras se va agitando el mar y los pescadores, hombres y mujeres, cantando, recogen con gran esfuerzo una red pesadísima. Se nos prepara la conocida escena del naufragio del Burlador, en que amo y criado se acercan en un barquito en este caso. Primero pide socorro don Giovanni, seguido por el criado. Aquí se perciben unos ecos de El burlador de Sevilla y diferencias fundamentales en el carácter del protagonista. A pesar de eso, cuando está a salvo y le pregunta la pescadora Elisa quién es, tiene igual cuidado que Tenorio de conservar el anonimato que tanto le conviene. Contesta secamente: "un cavaliere"'. La joven se ha fijado bien en su aspecto aristocrático y, lo mismo que nuestra Tisbea y tantas otras campesinas, desconfía de los elogios del caballero y de sus promesas de amor eterno: "Se creder vi potessi..." (p. 9). Es ella, Elisa, y no la novia Aminta, que aquí falta como falta este personaje en muchas obras donjuanescas, quien le obliga a hacer el juramento que más adelante ha de llevar a su condena (p. 10). Una vez que él jura "al Nume ch'al Cielo, e al Mondo impera", hacerla su mujer, Elisa pregunta qué pasará si él no es fiel a su palabra. A lo cual contesta don Giovanni: "Cada un fulmin dal Ciel, e l'alma infida / Precipiti agl' abissi"' (ibid.). La contestación satisface las dudas de la joven; ella le da la mano, y la

de $1777-\mathrm{y}$ un posible estreno en Praga.

${ }^{7}$ Citamos en su totalidad la ficha: "Nunziato Porta / Il convitato di pietra o sia Il dissoluto Dramma tragicomico per musica da rappresentarsi nel' teatro Esterház nell estate l'anno 1781. / [Vienna? 1781] / 8 .55 p. 17 $\mathrm{cm}$. / Author named on p. [2]; erroneously attributed to Antonio de' Filistri da Caramondani. / Without the music by Vincenzo Righini. / No. 6 in v. 64 of a collection of Italian plays'.

${ }^{8}$ PorTa, op. cit., acto I, esc. 1, p. 7. En adelante las páginas citadas de esta obra se darán entre paréntesis en nuestro texto. Cf. la contestación de Tenorio a la pregunta del rey "¿Quién eres?": “¿Quién ha de ser? / Un hombre y una mujer", El burlador de Sevilla, Clásicos Castellanos, Madrid, 1932, p. 167. 
escena acaba con una canción lírica de su amor y las cualidade de él.

Lo demás del primer acto (escenas 3 a 12) lo ocupan princ palmente escenas relacionadas con Donn' Anna, el Commend: tore, Don Alfonso e, indirectamente, con Donn' Isabella y el dı que Ottavio. El autor ha preferido entrelazar a estos personaj، en un episodio a diferencia de Tirso que les dedica episodios ind viduales. Don Alfonso llega con la noticia de que el Rey desc honrar al Comendador con una estatua ecuestre que sirva de te timonio a los muchos servicios que ha prestado a la corona Además, nos enteramos de que el Rey piensa arreglar el matı monio de Donn' Anna con el duque Ottavio. Es evidente la anı patía que le tiene la dama a esta unión, actitud que evoca en lector o espectador las interpretaciones románticas de la ópera c Da Ponte-Mozart, empezando con E.T.A. Hoffmann, en que sospecha que Donn' Anna se siente atraída al asesino de su $\mathrm{p}$ dre. Se cree que aplaza su casamiento con Don Ottavio otro ai por ese motivo a pesar de que se entiende que lo quiere y de q ya está vengada al final de la ópera.

Otros detalles menores del primer acto recuerdan la comed española. La intención de Don Giovanni es raptar a Donn' Ans y seducirla. Con este fin logra conseguir entrada en la casa. I falta el toque de pedir la dama -en Tirso es Isabela- que traiga luz para iluminar la escena, esa luz amenazadora que ir vitablemente traiciona al hombre cuyas hazañas viles exigen abrigo de la oscuridad protegedora, luz que anuncia el fuego $\mathrm{q}$ irá aumentando hasta consumir al malhechor al final. Siguen ] bien conocidas escenas de la llegada del Comendador, el duc en que lo mata Don Giovanni y los votos de venganza.

Es en la segunda jornada, que gira alrededor de la figura la estatua y que sigue más o menos la estructura establecida $\mathrm{F}$ Tirso, donde mejor se revela el carácter de este don Juan. Es hombre que por un lado anda tras la búsqueda de la hermosu y por otro, se queja de su destino que le ha obligado a ser homi

${ }^{9}$ Posiblemente sea la primera estatua ecuestre del Comendador la Claude Deschamps, Sieur de Villiers, Le Festin de Pierre ou le Fils criminel, 16 1660. Sin embargo, ésta se construye después de la muerte del Comendac al igual que la estatua de Tirso. Thomas Shadwell nos ofrece otra esta ecuestre en The Libertine, 1676; pero lo más probable es que el episodio de F ta tenga su origen en la obra de Carlo Goldoni, Don Giovanni Tenorio ossz dissoluto (estreno 1736), en que D. Alfonso le cuenta al Commendatore , el Rey ha mandado levantar en su honor una estatua ecuestre (acto F, esc. 
da. Para tranquilizar su conciencia pasa toda la responsabilidad de su conducta a la mujer y a Eros: "Oh Donne all' huom funeste / Per la vostra beltà!. . . Crudo perverso Amor!" (p. 26). Ya a partir de la escena del cementerio Don Giovanni se siente acometido de pensamientos sombríos, anhela la paz y apenas le sostienen las piernas. Cuando da con Donn' Anna ante la estatua del Comendador, descubre el pecho y le pide a la dama que lo mate. Pero en seguida vuelve en sí, a su ser donjuanesco, y le habla a Anna del amor que siente; además, trata de disculparse por haber matado a su padre. Se ve que tratamos con un personaje bien distinto del don Juan orgulloso, atrevido, insolente de Tirso $^{10}$. A tal punto llega a rebajarse este Don Giovanni que le pide compasión a Anna y le asegura con sus lágrimas que está arrepentido. Tampoco tiene este personaje mucho que ver con el don Giovanni de Mozart, que aparecerá pocos años más tarde y que se niega tres veces a arrepentirse ante la estatua que se lo manda. Más bien es, como dice él mismo, "un sventurato Amante" (p. 30). A pesar de que Donn' Anna trata de mantenerse firme en su denuncia, los encantos del caballero triunfan, de lo cual él se da perfecta cuenta mientras va recargándolos. Sin embargo, aunque sus lágrimas y contricción aplacan la ira de Donn' Anna, ésta sigue constante en la necesidad de vengarse, y le dice a Don Giovanni al marcharse que su muerte o perdón dependen de la víctima, su padre.

El encuentro con la estatua del muerto deja al burlador aún más perplejo y turbado, con la sensación de que se le acerca el día de su fin. La figura de piedra no sólo ha afirmado con la cabeza que acepta la invitación a cenar, sino que, ante la insistencia de Don Giovanni en que quiere oírlo de su propia boca, contesta: "Si verrò" (p. 33) ${ }^{11}$. El protagonista está tan desorientado que se pregunta si será verdad todo lo que está ocurriendo: "Sogno? vaneggio?" Todos andan persiguiéndole: Isabella,

${ }^{10}$ Cf. la escena del cementerio en el Invitado de piedra (Kamenyi Gost) de Aleksandr Pushkin (eses. 3 y 4 ) en que D. Juan, disfrazado de cura, trata de consolar a Da. Anna y luego se revela.

${ }^{11}$ En El burlador la estatua no da señal ni pronuncia palabra en su primer encuentro con D. Juan en que éste le invita a cenar, aceptando así el desafío de la inscripción de la tumba de $\mathrm{D}$. Gonzalo. La primera vez que habla es cuando llega al banquete. Por su lado, el libreto de Porta sigue la tradición italiana que lo precede; es decir, desde la comedia de gran influencia titulada Il convitato di pietra, atribuida a Giacinto Andrea Cicognini y fechada alrededor de 1650 o poco antes. 
Elisa, Anna, el Commendatore. Siente que en la cabeza le ard€ un fuego y que una serpiente le va oprimiendo el corazón (p. 34) Más adelante, mientras espera a su invitado, le acosan tenebro sas premoniciones de muerte. Cuando por fin llega la estatua J el huésped ofrece todo lo que puede proveer Castilla, el Comen dador se niega a probarlo, y explica que aceptó la invitaciór y acudió a la cita sólo para invitar a su vez a Don Giovanni J a Arlechino a cenar con él. Sacudiendo pensamientos oscuros miedo y dudas, el caballero acepta la invitación con que le corres ponde su víctima.

Al llegar al cementerio a ser fiel a la palabra que le dio a muerto, Don Giovanni, igual que un antepasado del Convitat atribuido a Cicognini, se siente algo incómodo ante tanta mues tra de luto. Es más; el personaje de Porta se estremece cuand, el Comendador le invita a comer serpientes, áspides y sapos, pe ro se domina y participa del banquete. Entonces la estatua, comc en otras obras, le pide tres veces que se arrepienta. Don Giovan ni se niega las dos primeras veces, y al tercer "Penteti" contesta "Se sia vero che in Cielo / Sovra 1' huomo mortal vi sia poter / S' e giustizia lassù / Nelle viscere sue m' ascondi il suolo" (pp 50-51). Y, en efecto, lo traga la tierra.

Arlechino sigue la tradición establecida por Tirso y vuelvı para darles la noticia a los demás. La primera reacción de las víe timas es de incredulidad, pero se les recuerda que el Cielo no enseña que el hombre muere como vive, y se afirma clarament la moraleja de que al disoluto y al impío le llegará su castigo. Li última escena, parecida a la del texto que se creía de Cicognini tiene lugar en el Infierno, aunque el contenido es otro. En la obr: anterior el libertino pide misericordia, que le niegan los demo nios. Aquí, este personaje más perverso maldice el día que naci y les advierte a otros que del mal sólo nace el mal y el bien d $\epsilon$ bien. Hay un coro de Furias, y cae el telón mientras se venga ca da una de las víctimas de Don Giovanni.

Este breve resumen de la obra de Porta-Reghini puede servi de base para colocarla en la tradición italiana que desciende $\mathrm{d}$ Tirso, a la vez que se reconoce la presencia de aspectos de la com media dell' arte y de aquellas obras que destacan el papel cómic del criado a expensas del amo, en que nosotros no nos hemos d $\epsilon$ tenido.

El otro libretto que examinamos - Il convito de Filippo Liviq ni- no suele clasificarse como obra donjuanesca, aunque sí figu ra en las bibliografías sobre el tema por el supuesto parecido dt 
título con Il convitato di pietra. Nuestra intención no pasa de querer aclarar algunas dudas y repasar ciertas aproximaciones al tema del don Juan que pueden sugerir una relación, por tenue que sea, con la leyenda.

Se sabe que Domenico Cimarosa compuso música para Il convito, pero no se ha podido confimar que lo haya hecho para ninguna obra titulada Il convitato di pietra. Tanto Singer como Weinstein hacen constar la duda, e incluyen el dato, éste en la sección de versiones musicales. Singer se aventura un poco más y alude a una anotación en la biblioteca de la Universidad de Harvard que incluye L'impresario in angustie, ed Il convitato di pietra: Farse per musica, con una segunda parte que lleva el subtítulo de Don Giovanni ossia Il convitato di pietra (Milano, 1789) ${ }^{12}$.

De hecho, en la colección de raros de la Houghton Library se encuentran Il convito y dos ediciones de diversas representaciones del Impresario in angustie, acompañadas éstas del Convitato di pietra $^{13}$. Al cotejar las fichas con los dos textos queda aclarado que las obras para las cuales compuso música Cimarosa no tienen que ver con nuestro tema. Corresponden al Impresario... y al Convito. La "segunda parte" a que se refiere Singer es, en realidad, otra obra, Don Giovanni ossia Il convitato di pietra, para la

${ }^{12}$ Singer, op. cit., p. 71; WeInStein, op. cit., p. 207.

${ }^{13}$ La ficha del catálogo reza: “[Diodati, Giuseppe Maria] / L'impresario in angustie, ed II convitato di pietra, farse per musica da rappresentarsi nel teatro alla Scala l'autunno 1789. / In Milano, per Gio. Batista Bianchi regio stampatore, colla permissione. / [1789] / «Il convitato di pietra» by Giovanni Bertati has separate signatures, separate paging and divisionai title: $\mathrm{D}$. Giovanni ossia II convitato di pietra. / Without the music by Domenico Cimarosa (L'impresario in angustie) and Gazzaniga (Il convitato di pietra)'. La otra ficha remite a la colección de comedias italianas, también bajo la autoría de Diodati. Lleva la asignatura $1 \mathrm{C} 7$. A100. B 750, t. 78 y reza: ' $L$ 'impresario in angustie, dramma per musica di un atto da rappresentarsi sul regio teatro di via della Pergola la primavera del MDCCXII. In Firenze MDCCXII. Nella stamperia Albizziniana da S.M. in Campo. "Don Giovanni Tenorio o sia Il convitato di pietra, dramma d'un atto solo" di Giovanni Bertati'. Parece confirmarse Diodati como autor en el Catalogo delle Opere Liriche Pubblicate in Italia de Aldo Caselli (Leo S. Olschki, Firenze), 1969, pp. 110-111. Se indica que el texto de L'impresario es de G. M. Diodati; da como fecha 1786 y lugar, Nápoles. Más adelante (pp. 196-197) aparece la obra de Diodati con música de Gazzaniga, 1789 en Ferrara y con música de Valentino Fioravante (pp. 164165), 1789. Además figura L'impresario sin autor con música de I. Ricci en Nápoles en 1822. Véase también Singer y Weinstein s.v. Giuseppe Gazzaniga, pp. 92 y 208 respectivamente; bajo Bertati: Singer, p. 52; y bajo $\mathrm{Da}$ Ponte: pp. 150 y 208 respectivamente. 
cual compuso la música Giuseppe Gazzaniga. La confusión y la dudas respecto al Impresario. . y al Convitato. . . arrancan del he cho de que esas dos obras se publicaron juntas muchas veces er el siglo XviII. Mientras las fichas declaran a Bertati libretista di la obra sobre don Giovanni, eso no se puede confirmar en los tex tos de Houghton. En ambos casos se lee después del reparto úni camente que la música es de Gazzaniga y no dicen nada sobr el poeta, como ocurre con el Convitato de Porta (véase supra). Esı justifica la duda de Singer sobre el autor, que nosotros tampocı podemos resolver; sólo podemos conjeturar que esa informaciór se encuentra en otra edición ${ }^{14}$. La pequeña confusión de Singe en cuanto a los títulos se explica por el hecho de que los mismo textos traen un título en la portada - Don Giovanni ossia Il convitat di petra - y otro en la primera página - Il convitato di pietra, part unica ${ }^{15}$.

Volviendo nuestra atención ahora al Convito de F. Livigni encontramos también dos entradas que son las fichas para texto de dos representaciones distintas. El libreto de este "dramm giocoso per musica" que parece tener prioridad, el del Teatro $\dot{c}$ San Samuele de Venecia, es el de la función que tuvo lugar es 1782 durante Carnaval. El texto está en el tomo 93 de la colec ción ${ }^{16}$. La fecha de publicación que trae -MDCCLXXXIpuede indicar que la obra se imprimió antes de que tuviera luga la función de principios de febrero del año siguiente, siempre qu no sea errata. La misma obra se anuncia para la primavera d 1782 en el teatro de la Via della Pergola, y aparece en el tom 79 de la colección ${ }^{17}$. Estas dos presentaciones en Florencia y Ve

\footnotetext{
${ }^{14}$ Kunze, op. cit, también pone a Bertati como libretista de la obra c Gazzaniga, pp. 33 ss.

${ }^{15}$ En otra biblioteca de Harvard, la Loeb Music Library, hemos dad con la partitura de una versión francesa de L'impresario in angustie ou Le directi dans l'embarras: opera bouffon en deux actes. Musique du Sgor. Cimarosa. Parolt françaises par Mr. ${ }^{* * *}$, Paris, Sieber [1788?].

${ }^{16}$ Asignatura 1C7. A100. B750, t. 93. Copiamos la ficha: "Il convits dramma giocoso per musica di Filippo Livigni da rappresentarsi nel nobi teatro di San Samuele il carnovale dell'anno 1782. / In Venezia, MDCCLXXX Presso Modesto Fenzo. Con li debite permissioni / $8^{\circ} .56$ p. $17.5 \mathrm{~cm}$. / Witl out the music by Domenico Cimarosa. / $\mathrm{N}^{\circ} .5$ in vol. 93 of a collection . Italian plays". Tras el reparto se lee: "La musica è del Signor Domenico C marosa, Maestro di Cappella Napolitano, ali attuai servizio di S. M. il Re d Ile due Sicilie".

${ }^{17}$ Asignatura 1C7. A 100. B 750, t. 79. El texto de la ficha no indica si de Livigni: "Il convito, dramma giocoso per musica da rappresentarsi nel $r$
} 
necia deben agregarse a las mencionadas por Singer (p. 71), que dice haber visto en la Library of Congress (Wash., D.C.) el manuscrito original de la función de Londres, 1782.

En cuanto al texto de $I l$ convito, hay que decir de entrada que la invitación del título se refiere a un banquete festivo y no a una cena fúnebre ofrecida por un don Giovanni o por un invitado de piedra. La acción gira en torno a una comedia de errores en que Massimo, tipo burdo y anti-donjuanesco, desea enamorar a todas las mujeres o, mejor dicho, desea que todas ellas se enamoren de él y que juren ellas que así es. En realidad, la cosa no pasa de jactarse Massimo de lo mucho que le quieren las damas. En un micro-catálogo nombra a varias de las jóvenes:

\author{
Donna Giulia, Donna Flora, \\ Donna Porzia, e Giacomina \\ Siora Marzia Pizzibotti \\ Donna Flavia Ragagliotti \\ Si piccò Madama Sarta, \\ Venne in tempo Donna Marta, \\ Ma scoperto un paradosso... (jor. $1^{\text {a }}$, esc. 9)
}

No es Massimo el único aspirante a Tenorio, ya que Lampò, el huésped no invitado, también desea más mujeres de las que se le ofrecen, y se tiene que conformar con apagar su "sed de amor" nada más que con tres. A pesar de la lección que le da Lampó a Massimo sobre cómo enamorar a mujeres de diversas nacionalidades, no se les puede considerar ni a ellos ni al Conde - el otro personaje masculino- grandes seductores ni libertinos.

Sin embargo, la obra posee algunos detalles que evocan aspectos de la leyenda del convidado de piedra tal como se conoce en la literatura donjuanesca. Alfonsina, viuda caprichosa perseguida por Massimo y el Conde, alude repetidas veces a su marido difunto, Barbalò, cuya presencia se siente palpablemente, por lo tanto, a lo largo de la obra. Hasta desea que le pongan el retra-

gio teatro di via della Pergola nella primavera del MDCCLXXXII. / In Firenze MDCCLXXXII. Con Lic. de' sup. Si vende da Giovanni Risaliti Stampatore dirimpetto ai pp Filippini. / 8 ${ }^{\circ}, 56$ p. $17 \mathrm{~cm}$. / Device of the Accademia degli Immobili on t.-p. / Without the music by Domenico Cimarosa. $\mathrm{N}^{\circ} .5$ in v. 79 of a collection of Italian plays". Tras el reparto se lee la misma aclaración sobre la música que la ficha anterior, sólo que aquí al músico se le denomina el "Celebre Signor Domenico Cimarosa". 
to del difunto frente a ella en el banquete, y es precisamente a ese retrato que se encomienda Massimo antes de un duelo con su rival. Más tarde Lampò, disfrazado del fantasma de Barbalò, impide que Massimo y el Conde hagan su duelo con pistolas. Al ver al "fantasma" y al criado, Checco, también disfrazado de Barbalò, Eleonora, dama, y Lisetta, criada de Alfonsina, gritan y piden socorro. Como aquí prevalece la comicidad, cada uno de los "espectros" le pide al otro que se descubra y pregunta quién es. Checco, representando a Barbalò, dice que su "esposa" debe casarse con Massimo; de lo contrario, el palacio será destruidc por un gran incendio. El lector recordará no sólo el incendio del convento en la obra de Zorrilla, sino la escena de Tirso en que la mansión de los Ulloa se ilumina con el resplandor de las luces que se encendieron en cuanto gritó doña Ana porque don Juan acababa de matar a su padre. En El.burlador de Sevilla el tema de] fuego se destaca también en la pasión ardiente de Tisbea y en su vergüenza abrasadora cuando Tenorio la engaña y deja ardiendc su choza al escaparse. Mientras en Tirso acaba el acto con el es. fuerzo de los pescadores por apagar el incendio de la casita de Tisbea y con un sentimiento de suerte funesta, el primer acto de Il convito se cierra alegremente con el canto de todos al palacic que arde. En cuanto a Barbalò, la única alusión directa a él er lo que sigue se hace en la segunda escena del segundo acto, cuan. do los congregados ofrecen sus brindis y el Conde hace un brin. dis al difunto.

En las dos escenas de la segunda jornada que tienen lugar er el jardín aparecen, además de los "fantasmas", varias estatuas incluso una de Cupido. Al igual que lo ocurrido antes con lo: fantasmas, hay dos personajes masculinos que se disfrazan de es. tatuas y se colocan en el jardín dieciochesco. En la primera df esas escenas hablan los dos en sílabas y sonidos ininteligibles, pe ro logran asustar a Massimo y a Alfonsina cuando les dicen qu la muerte les espera si se casan; cosa que hacen porque uno di los disfrazados aspira él mismo al matrimonio con Alfonsina. Po: muy tenue que sea la relación entre esta escena con alguna de Burlador, sí podemos ver en ella la parodia de una tradición co mún que comparte con la escena del cementerio de Tirso. Er cambio, la otra escena de estatuas no tiene parentesco alguno cor la obra española. Está poblada de cuatro o cinco figuras enmas caradas, recurso que sirve para juntar las parejas al final. Es evi dente que estas efímeras alusiones a un tema bien conocido d la época no son más que sugestiones muy tenues y paródicas 
sólo dignas, a lo sumo, de mencionarse como posible derivado alegre del original.

Todo esto nos deja sin resolver otros posibles vínculos entre Cimarosa y el tema del convitato di pietra, y con el deseo de fijar con mayor precisión fechas y lugares de las primeras representaciones, además de relaciones claras y seguras entre compositores y libretistas. Como se ve, don Juan y el convidado siguen engañándonos y proporcionando material para múltiples interpretaciones, innovaciones y divagaciones. Ya lo dijo Yves Florenne hace años en un artículo al hablar de "Don Juan á n'en jamais finir"'18.

Denah Lida Brandeis University

${ }^{18}$ La Table Ronde, 155 (1960), 149-156. 
